\title{
Benign Fibro-Osteo-Cemental Lesions of the Craniofacial Complex - A Review
}

\author{
Dr.Mrs.Jyoti S.Khedgikar ${ }^{1}$ \\ Professor and Head,Dept.Of Oral Pathology,CSMSS Dental College \& Hospital,Aurangabad (M.S.).
}

\begin{abstract}
Benign fibro-osseous lesions of the craniofacial complex are grouped together under the generic term 'Fibro-osteo-cemental lesions'. These lesions are characterized by pathologic ossifications and calcifications in association with a hypercellular fibroblastic marrow element. The concept of the 'Fibroosseous lesions' of bone has evolved over several decades to include two major entities : Fibrous dysplasia and ossifying fibroma. Other dysplastic bone lesions of the craniofacial complex include florid osseous dysplasia, focal cemento-osseous dysplasia and periapicalcemental dysplasia.
\end{abstract}

Keywords: Benign Fibro-osseous lesions, Fibrous dysplasia, ossifying fibroma, Osteitis deformans, Cementoma.

\section{Introduction}

Benign fibro-osseous lesions of the craniofacial complex are grouped together under the generic term 'Fibroosteo-cemental lesions'. These lesions are characterized by pathologic ossifications and calcifications in association with a hypercellular fibroblastic marrow element. The current classification includes neoplasms, developmental dysplastic lesions an inflammatory/reactive processes.

Miro S Mekek (1985) in his study of fibro-osteo-cemental lesions stated that, the only cells occurring in craniofacial jaw bones, which have ability to produce these lesions are osteoblasts, cementoblasts and fibroblasts.

The concept of the 'Fibro-osseous lesions' of bone has evolved over several decades to include two major entities : Fibrous dysplasia and ossifying fibroma. Other dysplastic bone lesions of the craniofacial complex include florid osseous dysplasia, focal cemento-osseous dysplasia and periapicalcemental dysplasia.

The histologic classification of fibro-osseous lesions of the bone has been widely debated and remains a source of controversy. The major controversies discussed are-

1. Identifying tissue origin

2. Distinguishing ossifying fibroma from fibrous dysplasia.

3. Seperating ossifying fibroma into meaningful pathologic categories and 4. Identifying and predicting agreesive lesions histologically. Hammer et al and Mekek advocated the periodontal origin of ossifying fibroma. The periodontal ligament has been shown to be capable of producing cementum and the osteoid, both of which are characteristically found in ossifying fibroma. Krausanetal and Spjut et al, however postulated that, primitive mesenchymal cells in areas such as the ethmoid bone and long bones may produce cementum at sites distant from odontogenic tissue. Eversole et al, suggested that all the lesions in the group i.e. ossifying/cementing fibroma and cemento-ossifying fibroma should be referred as ossifying fibroma and described the following classification of benign fibro-osseous lesions of craniofacial complex.

Classification :

I Bone dysplasias :

a) Fibrous dysplasia

i) Monostotic

ii) Polyostotic

iii) Polyostotic with endocrinopathy (McCune-Albright)

iv) Osteo-fibrous dysplasia.*

*Osteofibrous dysplasia is found in the tibia fibula only.

b) Osteitisdeformans

c) Pagetoid heritable bone dysplasias of childhood.

d) Segmental odontomaxillarydysplasias.

II Cemento-osseous dysplasias :

a) Focal cemento-osseous dysplasias

b) Florid cemento-osseous dysplasias.

III Inflammatory / reactive processes : 
a) Focal sclerosing osteomyelitis

b) Diffuse sclerosing osteomyelitis

c) Proliferative periostitis.

IV Metabolic disease : hyperparathyroidism.

V Neoplastic lesions : (Ossifying fibromas)

a) Ossifying fibroma (NOS)

b) Hyperparathyroidism jaw lesion syndrome.

c) Juvenile ossifying fibroma.

i) Trabecular type

ii) Psammomatoid types

d) Gigantiformcementomas.

Some investigators include giant cell lesions of bone in benign fibro-osseous lesions (BFOL). These lesions are not included by Eversole et al in their classification.

Second classification based on microscopic structures of fibrocellularcomponent and ossification variation is as follows :

I) Fibrous element variation.

- Homogeneous, plump monomorphic fibroblasts, hyper- cellularity, thin collegenfibers.

- Mature, hypocellular.

- $\quad$ Fasiculated, storiform.

II) Ossification (trabeculation) variation.

- Metaplastic woven bone

- Chinese / Hibrew figure trabeculae

- Lamellar bone trabeculae

- Osteoblastic rimming

- Mosaic resting and reversal lines

- Trabecular paralleling

- Cemental woven

- Cementalmicrolamellar

- Sharpey'sfiber fringe

- Droplet (Psammomatoid)

- Curvilinear conglomerates (Ginger root).

\section{Fibrous dysplasia}

It is a benign dysplastic process of altered osteogenesis that may occur within a single bone (monostatic) or multiple bones (polyostotic) associated with other anomalies and endocrinopathy i.e. called as McCune Albright's syndrome (MAS). The lesion of fibrous dysplasia shows elevated intracellular cAMP in bone marrow,osteo-proginator cells and these molecular changes probably initiate cell proliferation with differentiation defects.

It is a self limiting slow growing process that starts in childhoodand is usually diagnosed by age 20 years. A diagnosis of fibrous dysplasia should be done with clinicopathologic correlation. In these patients, swelling is unilateral and asymptomatic. It exhibits ill defined margins, blends into surrounding bone, and appears as a diffusely radio-opaque lesion with characteristic "ground glass" image.

In craniofacial complex, fibrous dysplasia is most commonly seen in maxilla and calvarium, whereas in the rest of the skeleton, it is seen most frequently in rib, femur and tibia. The process usually stabilizes during puberty, persisting in a nearly quiescent state indefinitely. When treatment is necessary to alleviate unacceptable facial deformity surgical recontouring, rather than complete excision is preferred. Regrowth of surgically contoured fibrous dysplasia is seen in approximately $25 \%$ of cases. Complete or partial excision with bone grafting has been recently used as a treatment.

Microscopically, fibrous dysplasia consists of relatively vascular and loose benign fibrous connective tissue stroma surrounding immature fibrillar or woven bony trabeculae. The stroma generally exhibits only low to moderate cellularity. The incompletely calcified bony trabeculae show some regularity in size and shows Chinese letter pattern gradually blending into normal surrounding bone. Osteoclasts are typically inconspicuous, and osteoblasts are scant showing the appearance of the tumour bone referred to as 'osseous metaplasia'. In later stages of the disease woven bone is replaced by lamellar bone trabeculae, extensive remodeling may result in a mosaic pattern of resting and reversal lines.

\section{Paget's Disease (Osteitisdeformans)}


This disease is named for Sir James Paget who presented an excellent description of this condition, characterized by simultaneous resorption and apposition of bone. Classic Paget's disease of bone is an osseous dysplasia with late onset. It is characterized by rapid turnover of bone resulting in osseous expansion with progressive skeletal deformities. Tubular bones show bowing and spinal curvature with vertebral collapse occurring in the later stages of disease.

Serum alkaline phosphatase level is markedly elevated. All bones of craniofacial complex can be affected to varying degrees. As facial and skull bone remodeling evolves, cranial nerve neuropathies are also seen, as a consequence of foramina narrowing with deafness being the predominant finding most of the time. In the early stages of the disease radiolucent 'coin' shaped lesions appear in the flat bone of the skull i.e. osteitiscircumscripta. Microscopically these are cellular fibro-osseous lesions with minimally calcified osteoid trabeculae exhibiting osteoblastic rimming with concomitant osteoclasticresorptive lacunae. Multinucleated cells are also found within the fibrocellular foci, without juxtaposition to the osseous elements. In addition, osteoclasts are numerous, larger than normal and have increased number of nuclei per cell. The bones of the maxillo-facial complex manifest a groundglasstrabecular pattern in the early stage disease yet with progression, diffuse sclerosis is seen radiographically, yielding the so called 'cotton wool' appearance of confluent nodular opacification.

Histologically, sclerotic craniofacial lesions show marked evidence of turnover, resting and reversal lines of lamellar compact and trabecular bone are prevalent and haphazardly arranged into a mosaic pattern. Another dental finding is generalized hypercementosis which is most advanced on molar and premolar teeth. There are many reports that have described viral particles and arrays in the osteoclastic cells of CPDB. Nucleic acid sequences and microbial antigens have been observed for paramyxo viruses (Measles in particular). Development of the osteosarcoma as well as giant cell tumour of both benign and malignant type is also noted. Treatment includes use of calcitonin, a parathormone antagonist produced by the thyroid gland which suppresses the bone resorption.

Diphosphonates are also used as they inhibit bone resorption and bone mineralization.

\section{Segmental odontomaxillary dysplasia}

A unique monostotic pathologic process that occurs in the maxilla is segmental odontomaxillary dysplasia. The lesion is confined to a single segment of the maxilla, usually involving the premolar and molar teeth and associated alveolar bone. Some would elect to exclude this entity from the classification of BFOLs based on the histology which is unique. Clinically teeth often fail to erupt and in the affected region, dental anomalies are encountered includingmalformed and misshapened teeth. There are defects in enamel also.Affected alveolar bone shows mild expansion and radiographicallynormal trabeculation is replaced by opacified streaks that resemble falling sleet. Microscopically, a fibro-osseous pattern is seen.

Both woven and lamellar irregular trabeculae are seen, with minimal, osteoblastic rimming. Small immature collegenfibers with mild increase in cellularity are also observed. In many areas, more dense sclerotic bone is seen. Cementicles and ovoid calcifications are also seen. The bone shows unique mosaic pagetoid resting and reversal lines, with prominent osteocyte hypercellularity characterized by multiple large lacunae.

\section{Cemento-osseous dysplasias}

The WHO definition of periapicalcemental dysplasia is a non-neoplastic lesion affecting the periapical tissues of one or more teeth. Focal cemento-osseous dysplasia (FCOD) and periapicalcemento-osseous dysplasia (PCOD) share the same histopathology and show similar, if not identical clinical profiles prompts one to consider that both are same entity with different locations.

\section{Focal cemento-osseous dysplasia}

This entity occurs predominantly in females with mean age in the mid-thirties and slightly higher distribution among African Americans.

Majority of the lesions are asymptomatic with an average size of $1.5 \mathrm{~cm}$. Mandible is the most frequent site of occurrence.

Radiographically, this condition evolves through three phases early, intermediate and late stages i.e. radiolucent, mixed radio-opaque-radiolucent pattern, with well-defined radiolucent rim around the radio-opacity and these lesions display diffuse radio-opacity often with ill defined borders.

\section{Florid cemento-osseous dysplasia}


When the lesions with radiologic and microscopic features similar to FCOD extend to two or more quadrants of the jaw, the disease is termed 'Florid cemento-osseous dysplasia'. In this lesion, mandible is affected bilaterally. Initially the lesion is asymptomatic.

Radiographically, this disease is characterized by multiple, confluent and non-expensile radio-opacities, often with a circumferential radiolucency. These lesions are most common in the mandibular molar/premolar area. Pain is the most frequent complaint, if patient becomes symptomatic. An odontogenic infection in teeth overlying lesional tissue may result in widespread infection evolving into an acute suppurative osteomyelitis with bony sequestration. FCOD in the primary stage histologically reveals multifragmented tissue component composed of vascular fibrous stroma with scattered osteoid trabeculae.

In the intermediate stage the vascular stroma becomes more fibrotic with additional formation of osteoidtrabeculae. Occasional cement like calcifications are also seen. In the late state corresponding to radiographically demonstrable opacification FCOD often displays verylittle fibrotic stroma with thick curvilinear trabeculae (ginger root pattern) or irregularly shaped cementum like masses.

\section{Inflammatory/Reactive processes}

Most infections of the jaws are odontogenic in origin. Both pyogenic and anaerobic bacteria (Streptococci,becteroides, actinomycesa) are responsible for acute, subacute or chronic osteomyelitis. These infections when suppurative may develop intraoral as well as cuteneoussinus tracts. The osseous lesions are destructive, showing typical 'moth-eaten' radiolucencies, owing to the osteoclasticresorptioninduced by the infectious agents and the host inflammatory response.

Anachoretic infections may also seed the jaws to cause osteolyticlesions, yet an odontogenic source of infection from dental caries is far more common.

Low grade infections may occur within the craniofacial bones, usually the mandible and initiate osteoblastic rather than osteoclasticactivity. These infections are caused by anaerobic organisms of low virulence. The host response to these low grade infections isreactive osteogenesis and imaging patterns show diffuse homogeneous opacification. Reactive proliferation of the periosteum is also noted. Focal sclerosing osteomyelitis (condensing osteitis), diffuse sclerosing osteomyelitis and proliferative periostitis are the inflammatory reactive processes which are also considered under the classification of fibro-osseous lesions.

\section{Hyperparathyroidism}

In primary and secondary hyperparathyroidism benign fibro-osseous lesions are reported among patients with renal osteodystrophy. Radiographically the jaws and facial bones show loss of normal trabeculation, replaced by a non-expansile diffuse groundglassopacification. Microscopically, a BFOL characterized by trabecular bone is seen.

\section{Ossifying fibromas}

Neoplasms with a fibro-osseous histology are represented by the ossifying fibroma group of lesions. These are neoplasms in true sense, exhibiting progressive proliferative capabilities with bony expansion showing well defined margins radiologically.

Ossifying fibromas include ossifying/cementifying fibromas not otherwise specified (NOS) and specific subtypes which include psammomatoid variant of ossifying fibroma, trabecular variant of ossifying fibroma and gigantiformcementoma.

\section{Ossifying/cementifying fibroma (NOS)}

This is the most common form of ossifying fibroma which occurs in the maxilla and mandible. It represents a neoplastic process that is typically painless, presenting with the expansion of the buccal and lingual cortices. In larger lesions it may expand the inferior aspect of the mandible with displacement of teeth. Radiographically, early lesion shows radioluscency due to presence of non-calcified osteoid.

Over time, tumours become progressively radio-opaque as more matrixcalcifies. Commonly, ossifying fibroma shows a pattern that may be indistinguishable from fibrous dysplasia, with small irregular trabeculae of osteoid that are typically rimmed by osteoblasts. The stromal element is hypercellular and the fibroblastic cells are devoid of atypical cytologic features. Early formative tumour shows woven bone pattern, however when the lesion matures, osteoblastic rimming is seen. There are irregular trabeculae which are often lamellar. In cementifying form, typical osseous trabeculae are seen in addition tocemental structures, which are ovoid or droplet in shape. These ovoid calcifications resemble normal cementicles that are present in the periodontal ligament. Calcified material of different types is found in ossifying fibromas. Eversole (1985) described it as follows:

1. Osseous product - consists of woven / lamellar trabeculae.

2. Spheroid/curvoid product - either non-polarizable or exhibiting a Sharpey’sfiber like fringe. 
3. Dystropic appearing calcification - seen with a spheroidal configuration within a fasciculated connective tissue stroma.

4. Anaestomosing curvilinear trabeculae - exhibiting microlamellar or quilted patterns, when viewed with crossed polars. This feature observed in normal root cementum is known as 'Birefringent Property'. Juvenile ossifying fibroma or juvenile aggressive ossifying fibroma

includes two distinct clinicopatholoigc entities.

1. Trabecular juvenile ossifying fibroma (Tr JOF).

2. Psammomatoid Juvenile ossifying fibroma (PsJOF).

Tr JOF is more common in maxilla and mandible, while PsJOF is predominantly seen in extragnathiccraniofacial bones. Regardless of the variant form of ossifying fibroma NOS, the behaviour and natural history appears to be the same. The lesion grows slowly and expands cortices. Simple curettage and enucleation are generally sufficient for a cure. Recurrences are uncommon.

\section{Gigantiformcementoma}

An extremely rare form of ossifying fibroma is multifocal with tumours that are often massive. This lesion arises during childhood and progresses to cause facial deformity during early adult years. It can affect both the jaws and may arise in two, three or all four quadrants. Radiographically, marked expansion is seen with a radiolucent mass containing floccular calcifications. Microscopically benign hypercellularstroma, with monomorphic appearing fibroblasts and mature collegenfibers is seen. Mitotic figures are absent. Dispersed throughout are ovoid, often laminated, psammomatoidcalcifications, which are larger in size. Under polarized light, Sharpey'sfibers are seen to project radially from the larger spheroidal deposits that resemble cementicles normally seen in PDL.

Treatment for gigantiformcementoma is resection with immediate or staged reconstruction.

\section{Discussion}

There has been considerable controversy concerning the nosology of benign fibro-osseous lesions because of its varied histomorphologicvariations, in patterns of stroma and bone. Sometimes some similar or even identical microscopic features can be shared among two or more entities. R.Eversole et al classified these diseases according to pathogeneticmechanism, but as mentioned the most practical approach is to consider BFOL in the context of combined clinical, radiologic and pathologic characteristics. Age, sex and race are variables that carry weight in the formulation of final diagnosis. After the evaluation in detail, some of the cases, though, unquestionably belonging to the fibro-osteo-cemental group of lesions,could not be defined in terms of the WHO classification with complete certainty. However, when all the clinical, radiological and histological criteria were considered, the groups emerge, which are not included in the WHO classification, despite the fact that, they were both biologically and morphologically characteristic.

In view of these diagnostic discrepancies as R.Eversole said it seemed logical to embark on a synoptic evaluation of fibro-osteo-cementallesions of the craniofacial jaw bones considering its embryological,anatomical, clinical, radiological and histological origin.

\section{References}

[1]. M.Mekek : Clinical pathology of fibro-osteo-cemental lesions in the craniofacial and jaw bones - A new approach to differential diagnosis (S.Karger A.G.: 1983 Edn.).

[2]. Waldron CA, GiansantiJS : Benign fibro-osseous lesions of the jaws, a clinical radiologic, histologic review of sixty-five cases. II Benign fibro-osseous lesions of periodontal ligament origin. Oral Surgery, Oral medicine, Oral Pathology, 1973; 35 : 340-50.

[3]. R.Eversole, Lan SU, Samir Emofty :Benign fibro-osseous lesions of the craniofacial complex - A review. Head \& Neck Pathology, $2008 ; 2: 177-202$.

[4]. Joseph A Regezi : Fibro-ossseous lesions of the facial bones. Modern Pathology 15(3) : 331-41, 2002.

[5]. Waldron CA, GiansantiJS : Benign fibroosseous lesions of the jaws : A clinical, radiologic, histologic review of sixty five cases.

[6]. Oral Surg, Oral Med, Oral Pathol 1973; 35, 190-201.

[7]. Eversole LR, Leider AS, Nelson K : Ossifying Fibroma : A clinicopathologic study of sixty four cases. Oral Pathol 60: 505-11, 1985. 